\title{
Worlds and Antiworlds
}

\author{
Alexey V. Klimenko, Vladimir A. Klimenko \\ Physics Department, Chelyabinsk State University, Chelyabinsk, Russia \\ Email: alklimenko@gmail.com
}

Received 22 June 2014; revised 15 July 2014; accepted 8 August 2014

Copyright (C 2014 by authors and Scientific Research Publishing Inc.

This work is licensed under the Creative Commons Attribution International License (CC BY).

http://creativecommons.org/licenses/by/4.0/

c) (i) Open Access

\begin{abstract}
In the framework of the theory of the gravitational field, which distinguishes between particles and antiparticles, it is shown that even in the Early Universe its disintegration occurred into areas consisting of baryons (worlds), and areas consisting of antibaryons (antiworlds). It is hypothesized that astronomers have observed worlds and antiworlds for fifteen years. They are, according to the authors, objects that can be seen as relatively bright spots against the almost uniform background of cosmic microwave radiation, having a characteristic angular size of quarter degree.
\end{abstract}

\section{Keywords}

\section{Cosmology, Particles, Antiparticles, Worlds, Antiworlds, Gravitational Field}

\section{Introduction}

The question of the structures of the Universe, their origin and evolution is one of the central problems in cosmology [1]-[3].

In the theories that describe the growth of the structures, their evolution is calculated under the given initial conditions. Usually the theories are based on the general theory of relativity (GTR), which does not distinguish between particles and antiparticles. The difference between the known theories of the evolution of the structures consists in the choice of initial conditions. This choice is largely arbitrary. In the fundamental monograph [1] it is noted: "What caused the occurrence of the primary perturbations, what is their nature-there exist only vague guesses about it”. Because of the uncertainty of the knowledge of the initial perturbations, different hypotheses about them are considered; see [1] [3].

In present paper, it is assumed that the initial perturbations in the Universe are connected with its disintegration into worlds and antiworlds.

A beautiful idea of a charge-symmetric Universe and the initial perturbations associated with its disintegration into the fields of matter and antimatter was considered by Omnes [4]. It has been rarely discussed until now. It is believed that this idea is difficult to substantiate in the framework of general relativity, and it does not agree 
with the observations. In [1] it is noted that with all the beauty of the conception of the Omnes theory it encounters such difficulties which compel one to abandon the pattern of the evolution of the Universe proposed by him. We will note some of these difficulties which are believed to be "killing" idea of the symmetry of the Universe with respect to particles and antiparticles.

According to some calculations carried out in the framework of general relativity, even in the Early Universe the whole symmetric world of uniformly mixed particles and antiparticles had to be annihilated. In the standard cosmological model $\Lambda C D M$, based on general relativity, the possibility of "survival" of appreciable amount of matter and antimatter later than $10^{-3}$ seconds after the "Big Bang" is entirely ruled out (see, for example, [1] $[3])$.

Observations show that the Universe is filled with matter, but there is no antimatter in appreciable quantities. In order to explain this observational fact they assume that in the Early Universe, as a result of a spontaneous symmetry breaking in reactions producing particles and antiparticles, appeared for every billionth pairs of baryons and antibaryons arose approximately one "extra" baryon (see, for example, [1] [3]). In the era of the baryon-antibaryon annihilation, the whole of their symmetric part extinct preserved only "extra” baryons.

The theory of Omnes could be agreed with the observations, if there existed a regular mechanism of the separation of particles and antiparticles. But there is no such mechanism in this theory, since it is based on the standard theory of gravity, which does not distinguish between particles and antiparticles.

The authors [5] expressed doubts about the correctness of the fundamental hypotheses of standard general relativity that gravity does not distinguish between particles and antiparticles. An alternative theory of gravity, in which gravity distinguishes between particles and antiparticles, has been proposed. It is called two-signed gravity. In this theory, the idea of Einstein about the relationship of gravity and space-time geometry is preserved. It is considered that a charge-current tensor is the source of the gravitational field. The difference between this tensor from an energy-momentum tensor, which is the source of Einstein's gravity, is that the same type of contributions of particles and antiparticles are taken with opposite signs, the consequence of this assumption the presence of an antiattraction between particles and antiparticles (for details see [5]).

In two-signed gravity it is assumed that the Universe contains an equal number of particles and antiparticles immersed in a gravity-neutral vacuum form of matter. It is considered that the Universe, as a whole, is a gravityneutral.

In this paper, the present, we show that within two-signed gravity, a beautiful idea of the origin of structures in the early Universe as a result of its disintegration into worlds and antiworlds finds a natural substantiation. The disintegration is a result of action of a regular mechanism. It is caused by the presence of antiattraction between particles and antiparticles and attraction between particles and particles, antiparticles and antiparticles.

We use the following terms in the present work. Worlds and antiworlds are a region of space with an excess of baryons and antibaryons, respectively. According to the standard general relativity, there is a baryon asymmetry and the entire modern Universe is a World [1]-[3]. In this paper, we have shown that if the idea of the difference between particles and antiparticles in gravity is correct, then already in the early epochs of the Universe was split up into an infinite number of worlds and antiworlds and our World only one of the worlds.

In the framework of the gravity-neutral universe, the problem of initial perturbations is considered without any arbitrary assumptions. We believe that thermal fluctuations are initial perturbations are. This removes the uncertainty in setting the initial conditions, which took place in other theories of the origin of structures in the Universe.

\section{Stratification of the Universe into Worlds and Antiworlds}

We believe that the space environment consists of three components: vacuum forms of matter, matter and antimatter.

The modern composition of matter is as follows: electrons $(e)$ protons $(p)$, neutrons $(n)$, neutrinos $\left(v_{e}, v_{\mu}\right.$, $v_{\tau}$ ) weakly interacting "dark particles" $(D)(D$-dark), photons $(\gamma)$. The nature of weakly interacting $D$-particles is currently not quite understood [2] [3]. In literature they usually use a notation in the form of abbreviation WIMPs ( $W$-Weakly, $I$-Interacting, $M$-Massive, $P$-Particles) for the dark matter particles. We believe that the Universe is symmetric with respect to particles and antiparticles. The composition of antiparticles is: $\bar{e}, \bar{p}$, $\bar{n}, \overline{v_{e}}, \overline{v_{\mu}}, \overline{v_{\tau}}, \bar{D}, \bar{\gamma}$.

The present paper does not consider dark energy, since it is not gravitationally neutral. We believe that a va- 
cuum form of matter is a gravitationally neutral matter [5]. The equation of the state of that matter is: $P_{V}=-(1 / 3) \varepsilon_{V}$.

In the framework of two-signed gravitation, it is naturally assume that Universe is not only electroneutral, but also gravitationally-neutral too.

Under temperatures much higher than the threshold temperatures necessary for the creation of $D, \bar{D}, p, \bar{p}$, $n$ and $\bar{n}$ particles $\left(T \gg 10^{13} \mathrm{~K}\right)$ were going reaction of their creation and annihilation in the Universe at a high rate. In the early universe, their numbers was by nine-ten orders of magnitude larger than the particles $D, p$ and $n$ in the modern Universe.

Originally, the space environment was weakly heterogeneous. In it, there were thermal fluctuations. Their amplitude was different for different scales. In volumes containing $n$ particles/antiparticles, the initial amplitude of thermal fluctuations of $\delta n / n$ was at the level of $\sim 1 / \sqrt{n}$ [6]. In the expanding Universe, there acted a mechanism ensuring a regular growth of these fluctuations. According to two-sign gravity, the separation mechanism of initially uniformly intermixed particles and antiparticles in the early Universe consisted in the following. The initial thermal fluctuations of higher density of particles created local gravitational fields. They were dragged particles into these fluctuations and pushed antiparticles out of them. This created a regular growth of the fluctuations considered. Symmetric process took place in the increased density fluctuations of antiparticles. These fluctuations drew in antiparticles and pushed out particles. The growth of fluctuations of matter and antimatter in the gravity-neutral expanding Universe occurred until intensive annihilation of matter and antimatter started as a result of its cooling. By the beginning of the era of the annihilation of baryons/antibaryons, the amplitude of fluctuations in the density of matter and antimatter stipulated by the difference in their concentrations reached values $\delta \rho / \rho \sim \delta \bar{\rho} / \bar{\rho} \lesssim 10^{-10} \div 10^{-9}$ in volumes containing $\approx 10^{88}$ particles/antiparticles. It was small, but by many orders greater than the amplitude of the initial thermal fluctuations. Choosing the initial fluctuations of this scale is associated with the idea of considering the observable part of the Universe, as a consequence of one of these fluctuations. After the completion of the annihilation only inconsiderable excesses of particles over antiparticles and antiparticles over particles, existing in the fluctuations at the beginning of the era of the annihilation "survived" in fluctuations. The Expanding Universe split into embryos of worlds and antiworlds. In the era of the annihilation of baryons and antibaryons, the overwhelming part of them was annihilated and turned into electrons, photons, and weakly interacting neutrinos and their antiparticles.

It is convenient to characterize the epochs of the evolution of the Universe by redshift $z$ corresponding to them. Formula $z=\left(a_{0} / a\right)-1$ determines the redshift of the era (please see, for instance [1]). Here, $a_{0}$ is the scale of the modern Universe, and $a$ is its scale in the period considered. The era of the intense annihilation of baryons/ antibaryons $\left(T \simeq 3 \times 10^{12}\right)$ corresponds to redshift $z_{a} \approx 10^{12}$.

After the completion of the annihilation of electron-positron pairs in the era of $z \lesssim 10^{9}$, the Universe finally split into worlds and antiworlds, immersed in an almost homogeneous, uniformly expanding relativistic component of the space environment.

\section{Small Perturbations in the Early Universe}

Within the framework of two-signed gravity [5], let us investigate the linear stage of instability as a result of development which the Universe is split into worlds and antiworlds.

We believe that in the absence of perturbations the gravitational field is absent. Matter and antimatter are uniformly mixed. There takes place an equilibrium of forces of attraction and repulsion. As a consequence of one a uniform expansion of the Universe takes place [5]. The dynamics of perturbations is studied by us in the co-moving frame. Because of the smallness of perturbations, we conduct our investigation in the Newtonian approximation. In this approximation, the equation describing the gravitational field takes the form [5]:

$$
\Delta \Phi=4 \pi G(\rho-\bar{\rho}),
$$

where $\rho$ and $\bar{\rho}$ are the density of matter and antimatter, respectively; $\Phi$ is the gravitational potential; $G \simeq 6.67 \times 10^{-8} \mathrm{~cm}^{3} / \mathrm{c}^{2} \mathrm{~g}$ is the gravitational constant.

We consider the epochs in which processes of the creation and destruction of baryons/antibaryons exactly balanced each other. This occurred under temperatures higher than $10^{13} \mathrm{~K}$. For these epochs, the equations describing the conservation of matter and antimatter can be written as 


$$
\begin{aligned}
& \frac{\partial \rho}{\partial t}+\operatorname{div}(\rho \boldsymbol{u})=0, \\
& \frac{\partial \bar{\rho}}{\partial t}+\operatorname{div}(\bar{p} \boldsymbol{\omega})=0,
\end{aligned}
$$

where $\boldsymbol{u}$ and $\boldsymbol{\omega}$ are the field velocities relating to matter and antimatter, respectively. Matter and antimatter are seen as two interpenetrating fluids. We take into account that there exists antiattraction between particles and antiparticles and we write the equations of motion for matter-antimatter in the following form:

$$
\begin{aligned}
& \frac{\partial \boldsymbol{u}}{\partial t}+(\boldsymbol{u} \nabla) \boldsymbol{u}=-\frac{1}{\rho} \nabla P-\nabla \Phi-v(\boldsymbol{u}-\boldsymbol{\omega}), \\
& \frac{\partial \boldsymbol{\omega}}{\partial t}+(\omega \nabla) \boldsymbol{\omega}=-\frac{1}{\bar{\rho}} \nabla \bar{P}+\nabla \Phi-v(\boldsymbol{\omega}-\boldsymbol{u}),
\end{aligned}
$$

where $P, \bar{P}$ is pressure in matter and antimatter, $v$ is the effective frequency of collisions of particles and antiparticles. As can be seen later, the friction between the countercurrents of particles and antiparticles substantially affects the process of the stratification of the space environment in the Universe on the worlds and antiworlds. As a consequence of this, it is taken into account.

Equations (1)-(5) are used to study the gravitational instability of the expanding gravity-neutral space environment. The investigation of the instability is carried out in the linear approximation.

In [5] the dynamics of a homogeneous isotropic gravity-neutral Universe is considered. It is shown that there occurred its uniform expansion. The changing of the scale of the Universe $a(t)$ is described by the equation

$$
a(t)=c t,
$$

where $c$ is the speed of light. Hubble's expansion of the Universe takes place. The Hubble parameter is:

$$
H(t)=\frac{1}{a} \frac{\mathrm{d} a}{\mathrm{~d} t}=\frac{1}{t} .
$$

Hubble's unperturbed expansion can be also described within the framework of Equations (1)-(5). From these equations, it follows that for the unperturbed parameters the following relations are met:

$$
\rho_{0}(t)=\bar{\rho}_{0}(t), \nabla \rho_{0}=\nabla \bar{\rho}_{0}=0, \nabla P_{0}=\nabla \bar{P}_{0}=0, \boldsymbol{u}_{0}(t)=\boldsymbol{\omega}_{0}(t)=H(t) \boldsymbol{r} .
$$

Using (8), we find from (1)-(5):

$$
\begin{gathered}
\frac{\mathrm{d} \rho_{0}}{\mathrm{~d} t}+3 H(t) \rho_{0}=0, \\
\frac{\mathrm{d} H}{\mathrm{~d} t}+H^{2}=0 .
\end{gathered}
$$

Integrating (9), (10), we obtain

$$
\rho_{0} a^{3} \sim \rho_{0} t^{3}=\text { const, } H(t)=\frac{1}{t}, a(t)=c t .
$$

Suppose that a small perturbation is imposed on to an expanding space environment consisting of uniformly mixed matter and antimatter. The uniform, but time-varying value $f_{0}$ of any of the parameters of the space environment gets perturbation $f_{1}$.

It is easy to show that Equations (1)-(5) in the linear approximation with respect to perturbations can be written as

$$
\begin{gathered}
\Delta \Phi_{1}=4 \pi G \rho_{0}(\delta \rho-\delta \bar{\rho}), \\
\frac{\partial \delta \rho}{\partial t}+H \boldsymbol{r} \nabla(\delta \rho)+\nabla \boldsymbol{u}_{1}=0,
\end{gathered}
$$




$$
\begin{gathered}
\frac{\partial \delta \bar{\rho}}{\partial t}+H \boldsymbol{r} \nabla(\delta \bar{\rho})+\nabla \boldsymbol{\omega}_{1}=0, \\
\frac{\partial \boldsymbol{u}_{1}}{\partial t}+H \boldsymbol{u}_{1}+H(\boldsymbol{r} \nabla) \boldsymbol{u}_{1}=-u_{s}^{2} \nabla \delta \rho-\nabla \Phi-v\left(\boldsymbol{u}_{1}-\boldsymbol{\omega}_{1}\right), \\
\frac{\partial \boldsymbol{\omega}_{1}}{\partial t}+H \omega_{1}+H(\boldsymbol{r} \nabla) \boldsymbol{\omega}_{1}=-u_{s}^{2} \nabla \delta \bar{\rho}+\nabla \Phi-v\left(\boldsymbol{\omega}_{1}-\boldsymbol{u}_{1}\right) .
\end{gathered}
$$

The following notations are met

$$
u_{s}^{2}=(\partial P / \partial \rho)_{0}=(\partial \bar{P} / \partial \bar{\rho})_{0}, \delta \rho=\rho_{1} / \rho_{0}, \delta \bar{\rho}=\bar{\rho}_{1} / \rho_{0} .
$$

Similar equations for the case of the homogeneous expanding space environment which only consists of matter are well-known. They are described, for instance, in [1] and [3]. The problem of small perturbations in the homogeneous isotropic expanding Universe in the newtonian approximation was first considered by Bonnor [7]. If we assume $\delta \bar{\rho}=0, \quad \bar{\omega}_{1}=0$ in Equations (12)-(16), then we obtain equations describing Bonnor's task. Our consideration is more general. It takes into account the difference in the gravity of particles and antiparticles. It is due to the difference between particles and antiparticles, according to two-signed gravity that a regular mechanism of the separation of the Universe into worlds and antiworlds existed in the Early Universe.

System (12)-(16) is a set of linear equations with time-dependent coefficients. We believe is as is usually assumed (please see, for instance, [1] [3]) that due to the expansion of the Universe, is a change of wavelengths making up the perturbation takes place, and they change similar to its scale $a(t)$. Given that, we use conversion [1]-[3]

$$
f_{1}(\boldsymbol{r}, \boldsymbol{t})=f_{1 \boldsymbol{k}}(t) \exp \left(i \frac{\boldsymbol{k} \boldsymbol{r}}{a t}\right) .
$$

This transformation enables one to replace operators of differentiation on space variables by algebraic operations and obtain a system of ordinary linear differential equations for the Fourier amplitudes of the $f_{1 k}(t)$. Taking (18) into account, we get from (12)-(16):

$$
\begin{gathered}
k^{2} \Phi_{1}=-4 \pi \rho_{0} a^{2}(\delta \rho-\delta \bar{\rho}), \\
\frac{\mathrm{d} \delta \rho}{\mathrm{d} t}+i \frac{\boldsymbol{k}}{a} \boldsymbol{u}_{1}=0, \\
\frac{\mathrm{d} \delta \bar{\rho}}{\mathrm{d} t}+i \frac{\boldsymbol{k}}{a} \boldsymbol{\omega}_{1}=0, \\
\frac{\mathrm{d} \boldsymbol{u}_{1}}{\mathrm{~d} t}+H \boldsymbol{u}_{1}+i \frac{\boldsymbol{k}}{a}\left(u_{s}^{2} \delta \rho+\Phi_{1}\right)+v\left(\boldsymbol{u}_{1}-\boldsymbol{\omega}_{1}\right)=0, \\
\frac{\mathrm{d} \omega_{1}}{\mathrm{~d} t}+H \boldsymbol{\omega}_{1}+i \frac{\boldsymbol{k}}{a}\left(u_{s}^{2} \delta \bar{\rho}-\Phi_{1}\right)+v\left(\boldsymbol{\omega}_{1}-\boldsymbol{u}_{1}\right)=0,
\end{gathered}
$$

In Equations (19)-(23), symbol $\boldsymbol{k}$ is omitted in the notation of the Fourier amplitudes in order to avoid cluttering the formulas by indexes.

Using (19)-(23), we obtain a system of equations that determine the evolution of density perturbations of matter and antimatter. They can be written as

$$
\begin{aligned}
& \frac{\partial^{2} \delta \rho}{\partial t^{2}}+2 H \frac{\mathrm{d} \delta \rho}{\mathrm{d} t}+v\left(\frac{\mathrm{d} \delta \rho}{\mathrm{d} t}-\frac{\mathrm{d} \delta \bar{\rho}}{\mathrm{d} t}\right)+\left(\frac{k^{2} u_{s}^{2}}{a^{2}}-4 \pi G \rho_{0}\right) \delta \rho+4 \pi G \rho_{0} \delta \bar{\rho}=0, \\
& \frac{\partial^{2} \delta \bar{\rho}}{\partial t^{2}}+2 H \frac{\mathrm{d} \delta \bar{\rho}}{\mathrm{d} t}+v\left(\frac{\mathrm{d} \delta \bar{\rho}}{\mathrm{d} t}-\frac{\mathrm{d} \delta \rho}{\mathrm{d} t}\right)+\left(\frac{k^{2} u_{s}^{2}}{a^{2}}-4 \pi G \rho_{0}\right) \delta \bar{\rho}+4 \pi G \rho_{0} \delta \rho=0,
\end{aligned}
$$

In the problem considered, the matter and antimatter occur symmetrically. In the co-moving reference system, 
the speeds of their movement in the gravitational field at each point are equal in magnitude but just opposite in direction. Consequently, the number of particles entering some volume is equal that of antiparticles leaving it and vice versa. The ratio between numbers of particles and antiparticles varies with space and time. At the same time, the total concentration of particles and antiparticles only changes over time. In addition, this change is due to the expansion of the Universe. Considering the above, we believe that as long as matter and antimatter are in a local thermodynamic equilibrium, the following relations hold true:

$$
\boldsymbol{u}_{1}=\boldsymbol{\omega}_{1}, d \bar{r}=-d r, \nabla P=0 .
$$

According to (26), the change of density perturbations in matter and antimatter occurs in concert. No pressure disturbance in the period considered is present. It append later in the cosmic medium, in the era of intense annihilation.

Considering relations (26), the set of Equations (24), (25) is reduced to one equation. It has the following form

$$
\frac{\mathrm{d}^{2} \delta \rho}{\mathrm{d} t^{2}}+2 H \frac{\mathrm{d} \delta \rho}{\mathrm{d} t}+2 v \frac{\mathrm{d} \delta \rho}{\mathrm{d} t}-8 \pi G \rho_{0} \delta \rho=0
$$

We use this equation for analyzing the growth of density fluctuations of baryons/antibaryons in the Early Universe.

\section{The Growth of Perturbations in the Early Universe}

The idea of the research discussed in the present paper is as follows. We believe that with the presence of density fluctuations $\delta \rho$ and $\delta \bar{\rho}$, in the cooling Universe under temperatures lower than $3 \times 10^{12} \mathrm{~K}$, not all baryons/ antibaryons where able to annihilate. In the fluctuations where there was a surplus of particles $(\delta \rho>0)$ some "extra" baryons and vice versa in the fluctuations where $\delta \bar{\rho}>0$ some "extra" antibaryons remained. The ratio between the number of baryons/antibaryons which were not annihilated in these fluctuations and the number of photons in them is approximately equal to value $\delta \rho$ in Era $T \simeq 3 \times 10^{12} \mathrm{~K}$.

As part of equation (27), we will explore the fundamental possibility of the growth of $\delta \rho$ and $\delta \bar{\rho}$ in the Early Universe, under temperatures $T \gtrsim 3 \times 10^{12} \mathrm{~K}$, up a level of $10^{-9}$. We assume that the initial level of perturbations $\delta \rho(0)$ and $\delta \bar{\rho}(0)$ was by many orders of magnitude less than $10^{-9}$. We think that fluctuations $\delta \rho(0)$ and $\delta \bar{\rho}(0)$ had a thermal origin. We are interested in fluctuations containing approximately $n_{0} \approx 10^{88}$ particles/antiparticles. In this case, the initial perturbation level of $\delta \rho(0)$ and $\delta \bar{\rho}(0)$ was at a level of $10^{-44}$ $\left(\delta \rho(0) \sim \delta \bar{\rho}(0) \sim 1 / \sqrt{n_{0}} \sim 10^{-44}\right)[6]$.

An increase of the amplitude of perturbations $\delta \rho$, which was approximately by thirty-five orders of magnitude (from initial thermal $\delta \rho(0) \sim 10^{-44}$ ) might have been ensured if their exponential growth had taken place, and its characteristic time $\tau$ had been about eighty times less than age $\tau_{a}$ of the Universe in the era of the intense annihilation by baryons.

According to the model of the homogeneous isotropic gravity-neutral Universe (please see [5]), uniformly it is expanding. Considering that this is the case, we conclude that the era of the annihilation of baryons/antibaryons occurred approximately about the time of

$$
\tau_{a}=\tau_{0} / z_{a} \approx 4.2 \times 10^{5} \mathrm{C}
$$

after the "Big Bang". Equation (28) takes into account that present age of the Universe $\tau_{0} \simeq 14 \times 10^{9}$ years, and the annihilation of baryons/antibaryons occurred under $z \approx 10^{12}$.

In the era preceding the annihilation of baryons/antibaryons, the space environment was at a thermodynamic equilibrium with radiation. Unperturbed energy density $\rho_{0} c^{2}$ for matter/antimatter in this era was of the same order as that of radiation. With this in mind, we believe that at $T \gtrsim 3 \times 10^{12} \mathrm{~K}$

$$
\rho_{0} \sim \sigma T^{4} / c^{2} \geq 10^{15} \mathrm{~g} / \mathrm{cm}^{3},
$$

where $\sigma$ is the Stefan-Boltzmann constant. A characteristic time corresponding to these densities

$$
\tau_{0}=\left(8 \pi G \rho_{0}\right)^{-1 / 2},
$$

is a part of Equation (27), and appears to be $\leq 2.4 \times 10^{-5} \mathrm{c}$. 
A characteristic time of the variation of unperturbed parameters is value $H^{-1}$. In the model of the uniformly expanding Universe, $H^{-1}=t$. In times preceding the annihilation of baryons/antibaryons $H^{-1} \lesssim 10^{5} \mathrm{c}$.

In the epoch that interests to us, effective time $v^{-1}$ between collisions of particles-antiparticles, with the exception, perhaps, of the earliest moments of the evolution of the Universe, was much less than that of the characteristic time of the variation of its unperturbed parameters. The consequence of this, we believe that $v \gg H$ and in Equation (27) do not does the impact of the second term on the growth of perturbations.

The increase of perturbations of densities of matter/antimatter, $\delta \rho$ and $\delta \bar{\rho}$, from $10^{-44}$ to $10^{-9}$ can be obtained if the characteristic time of growth approximately about one-eightieth of the lifetime of the Universe in the era of the annihilation of baryons/antibaryons, $t_{a} \approx 4 \times 10^{5}$. Assuming this to be correctly and comparing the characteristic values of the first and last terms in the Equation (27), we conclude that in the Early Universe, in the era of $z>10^{12}$, the influence of summand $\mathrm{d}^{2} \delta \rho / \mathrm{d} t^{2}$ was not essential.

In view of the above estimates, Equation (27) which describes the growth of perturbations $\delta \rho(t)$ in the Early Universe can be approximately written as

$$
2 v \frac{\mathrm{d} \delta \rho}{\mathrm{d} t}=8 \pi G \rho_{0} \delta \rho
$$

In the estimations, considering that $v \sim n_{0}, \rho_{0} \sim n_{0}$, where $n_{0}$ is the undisturbed concentration of particles/ antiparticles, we approximately reckon that

$$
\tau=\frac{v}{4 \pi G \rho_{0}},
$$

in the epochs that interest us remains constant. In this case, the solution of Equation (31) with initial condition

$$
\left.\delta \rho\right|_{t=0}=\delta \rho(0)
$$

takes the following form

$$
\delta \rho(t)=\delta \rho(0) \exp (t / \tau)
$$

Value $\tau$ defines the characteristic time of the growth of perturbations, $\delta \rho$. In order to ensure growth $\delta \rho$ of perturbations from the $10^{-44}$ for $t=0$ to $10^{-9}$ for $t \approx t_{a}$ it is necessary that time $\tau$ shall be as follows:

$$
\tau \approx \frac{1}{80} t_{a} \simeq 5 \times 10^{3} \mathrm{c}
$$

Taking (29) and (32) in consideration, we find that condition (35) is satisfied in case effective collision frequency in countercurrents of particles-antiparticles is $v \approx 4 \times 10^{12} c^{-1}$. This frequency is by many orders of magnitude lower than that of collisions of nuclear particles, assuming that their speed is near-light and their concentration is $\gtrsim 10^{39} 1 / \mathrm{cm}^{3}\left(\rho_{0} \gtrsim 10^{15} \mathrm{~g} / \mathrm{cm}^{3}\right)$. Apparently, this means that under these conditions $\left(\rho_{0} \gtrsim 10^{14} \mathrm{~g} / \mathrm{cm}^{3}\right.$, $T \gtrsim 10^{13} \mathrm{~K}$ ) matter was in a superfluid state.

These estimates show that in the Early Universe some conditions are under which could exists, growth of $\delta \rho$ to values approximately equal $10^{-9}$, by the beginning of the baryon/antibaryon annihilation, in the volume containing approximately $10^{88}$ particles/anti-particles. After the annihilation of baryons/antibaryons, the Universe split into regions containing only baryons and areas in which antibaryons alone remained. In each of the regions under consideration, there remained around $10^{79}-10^{80}$ units. At the same time, a baryon-to-photon and antibaryon-to-photon ratios are at the level of $10^{-9}$ in these areas. In order of magnitude, the number of particles in the established Universe and baryon-to-photon ratio observed in the world around us regarding this, please see, e.g. [1] [2].

In conclusion, we will note the following. The conditions of gravitational instability in the Early Universe, according to Einstein's gravity and two-signed gravity, differ cardinally. In the first case, they were not implemented [1]-[3] while in the second, as shown above, they were carried out with a large margin. The difference in the development of gravitational instability in these theories is due to two reasons. The first one is connected with a completely different character of the expansion of the Universe predicted by these theories. The second one is related to the presence of a mechanism of the separation of particles and antiparticles in two-signed grav- 
ity and the absence of this mechanism in Einstein's gravity.

\section{Discussion of Results}

The above-stated research points to the following possibility of the disintegration of the Universe into worlds and antiworlds.

In the Early Universe under $z>10^{12}$, there acted regular mechanism of growth of density perturbations due to the difference in the gravity of particles and antiparticles. Regions of increased density of particles attracted particles to itself and pushed out antiparticles. A symmetrical situation occurred in areas of increased density of antiparticles. They drew in antiparticles and pushed out particles. As a result of the action of this regular mechanism, both the density perturbations were growing.

The growth of fluctuations which initially contained about $10^{88}$ particles and antiparticles was considered. This choice of the number of particles and antiparticles in the fluctuations is associated with idea of considering the observable part of the Universe as one of these fluctuations. We believe that they had a thermal nature at first. In these fluctuations, the random initial excess of the number of particles over antiparticles in the perturbations of the same sign as well as the random initial excess of antiparticles over the number of particles in the perturbations of the other sign, it was at a level of $10^{-44}$ symmetrical to it.

In the co-moving frame of reference at any point in the gravitational field from arising fluctuations, the particles and the antiparticles were moving with an equal but oppositely directed velocity. There was an exponential growth of fluctuations. The rate of the growth was determined by the equilibrium of forces of gravity and friction forces in countercurrents of particles/antiparticles. The estimates adduced in paragraph 4 show that there is all reasons to believe that the values of density perturbations $\delta \rho$ and $\delta \bar{\rho}$ in these fluctuations could grow to a value of approximately $10^{-9}$ in the Early Universe by the beginning of intensive annihilation of baryons/antibaryons.

In the era of the annihilation of baryons and antibaryons, overwhelming part of them perished. Only nearly one baryon/antibaryon per billion survived. This value is observed in the Modern Universe for the baryon-tophoton ratio. According to an explanation proposed in present paper, this ratio determined by an amplitude of density perturbations of particles/antiparticles at the beginning of the baryon/antibaryon annihilation.

After the annihilation of baryons/antibaryons in the considered fluctuations around $10^{79}-10^{80}$ baryons/antibaryons remained. In one half of them only baryons survived, and in the other only antibaryons.

A characteristic scale of formed embryos of worlds and antiworlds at $z \lesssim 10^{12}$ was approximately $10^{6}$ light seconds. In this assessment it is take into account that the mass density of baryons/antibaryons in the era of annihilation fell by about nine to ten orders of magnitude and become approximately equal to $10^{5} \div 10^{6} \mathrm{~g} / \mathrm{cm}^{3}$. These embryos of worlds and antiworlds were "frozen" in to a relativistic expanding space environment, which consisted mainly of a vacuum form of matter, electrons, neutrinos, photons, and their antiparticles. In that era, the overwhelming part of the energy of the space environment was enclosed in its relativistic component.

At $\mathrm{z} \lesssim 10^{9}\left(T \lesssim 3 \times 10^{9} \mathrm{~K}\right)$ an annihilation of electron-positron pairs took place, and the Universe finally broke up into worlds and antiworlds. Their scale at that time was approximately $10^{9}$ light seconds.

Baryons $(p, n)$ and electrons in worlds, and antibaryons $(\bar{p}, \bar{n})$ and positrons in antiworlds, were in a thermodynamic equilibrium with radiation for a long time, up to $z \simeq 10^{3}\left(T \approx 3 \times 10^{3} \mathrm{~K}\right)$. The expansion law of worlds and antiworlds in the era varying from $z \simeq 10^{9}$ to $Z \simeq 10^{3}$, differed little from the law of expansion of the Universe as a whole. A slight speed difference in the expansion of the Universe and that of worlds/antiworlds was connected with the influence of local gravitational fields of latter on their dynamics. It resulted in that at $z \simeq 10^{3}$ the average density of the worlds/antiworlds was by $10^{-4} \div 10^{-5}$ orders greater than that of the space environment in the Universe.

According to the model of uniformly expanding Universe, the size of worlds/antiworlds in epoch of recombination $\left(z \simeq 10^{3}\right)$ was around $14 \times 10^{6}$ light-years $(\simeq 4.2 \mathrm{Mps})$. According to calculations (see, [5]) currently worlds and antiworlds should be observed as objects with an angular size approximately equal to quarter degree in the framework of a uniformly expanding Universe in such linear dimensions.

The observation of the fine structure of cosmic microwave background radiation shows that there is some slight variations on its uniform background (please see, e.g., [2] [8]). They testify to the existence of irregularities in the distribution of visible matter. In many places of the microwave background, spots with angular size approximately quarter degree are clearly observed. It is precisely these dimensions that worlds and antiworlds in 
the era of recombination in accordance with two-signed gravity must have. With this in mind, we believe that there is evenly reason to believe that these spots are worlds and antiworlds. Apparently, they are regularly distributed in space and are the largest structural elements of the Universe. We live in one of the worlds. We believe that the impossibility of seeing clearly the periodicity of the spatial distribution of worlds and antiworlds in full scale is associated with the "blocking" influence of inhomogeneities which occurred in our world much later.

According to two-signed gravity, worlds and antiworlds do not seek to converge and collide. They are "frozen" into a uniformly expanding space of the gravitationally-neutral Universe. Their characteristic modern size is approximately $14 \times 10^{9}$ light-years. Worlds and antiworlds repulse each other.

Changing the scale of the Universe, $a(t)$, is defined by formula (6). Dimensions $R(t)$ of worlds/antiworlds are changing approximately as the scale of the Universe $a(t)$. The difference in the rate of expansion of the gravitationally-neutral Universe and gravitating worlds/antiworlds is related to the influence of gravitational fields of the latter on their dynamics. Approximately one can assume that at $z \lesssim 10^{9}$ the worlds/antiworlds expanding as independent Hubble balls with almost uniform initial distribution of matter/antimatter in them.

\section{Conclusions}

The original idea of charge-symmetric Universe suggested by was first P. Dirac in 1933, in his Nobel Prize lecture. It is a pleasure for us to adduce a paragraph from this lecture.

"If we accept the view of complete symmetry between positive and negative electric charge so far as concerns the fundamental laws of Nature, we must regard it rather as an accident that the Earth (and presumably the whole solar system), contains a preponderance of negative electrons and positive protons. It is quite possible that for some of the stars it is the other way about, these stars being built up mainly of positrons and negative protons. In fact, there may be half the stars of each kind. The two kinds of stars would both show exactly the same spectra, and there would be no way of distinguishing them by present astronomical methods.”

Based on the research discussed in the present paper, there is every reason to assume that antiworlds exist away and the nearest of them are at a distance of approximately $14 \times 10^{9}$ light years. We believe that the possibility of distinguishing between worlds and antiworlds in observations exists and is associated with a difference in the behavior of particles and anti-particles in a gravitational field. We will note also that, possibly, antimatter can be produced in the World. Relativistic stages of the evolution of massive "stars" can happen on them at high rate processes of birth/annihilation of particles/antiparticles. In this regard, according to two-signed gravity, an effective separation of particles and antiparticles, as well as formation of antimatter from them can take place. The antimatter born in the vicinity of these "stars" can be emitted into space surrounding them. This must become apparent in the observations.

\section{References}

[1] Zel'dovich, Y.B. and Novikov, I.D. (1975) The Structure and Evolution of the Universe [in Russian]. Nauka, Moscow.

[2] Gorbunov, D.S. and Rubakov, V.A. (2008) Introduction to the Theory of the Early Universe. Hot Big Bang Theory [in Russian]. LKI, Moscow.

[3] Gorbunov, D.S. and Rubakov, V.A. (2010) Introduction to the Theory of the Early Universe Cosmological Perturbations. Inflationary Theory [in Russian]. KRASAND, Moscow.

[4] Omnes, R. (1971) Astronomy Astrophysics, 10, 228.

[5] Klimenko, A.V. and Klimenko, V.A. (2014) Gravitationally-Neutral Universe [The Article "Worlds and Antiworlds' Is Appendix for Article “Gravitationnaly-Neutral Universe']”.

[6] Kittel, C. and Kroemer, H. (1980) Thermal Physics. Freeman \& Co., New York.

[7] Bonnor, W.B. (1957) Monthly Notices of the Royal Astronomical Society, 117, 104-117. http://dx.doi.org/10.1093/mnras/117.1.104

[8] Hinshaw, G., Nolta, M.R., Bennet, G.L., et al. (2007) The Astrophysical Journal Supplement Series, 170, 377-408. http://dx.doi.org/10.1086/513698 
Scientific Research Publishing (SCIRP) is one of the largest Open Access journal publishers. It is currently publishing more than 200 open access, online, peer-reviewed journals covering a wide range of academic disciplines. SCIRP serves the worldwide academic communities and contributes to the progress and application of science with its publication.

Other selected journals from SCIRP are listed as below. Submit your manuscript to us via either submit@scirp.org or Online Submission Portal.
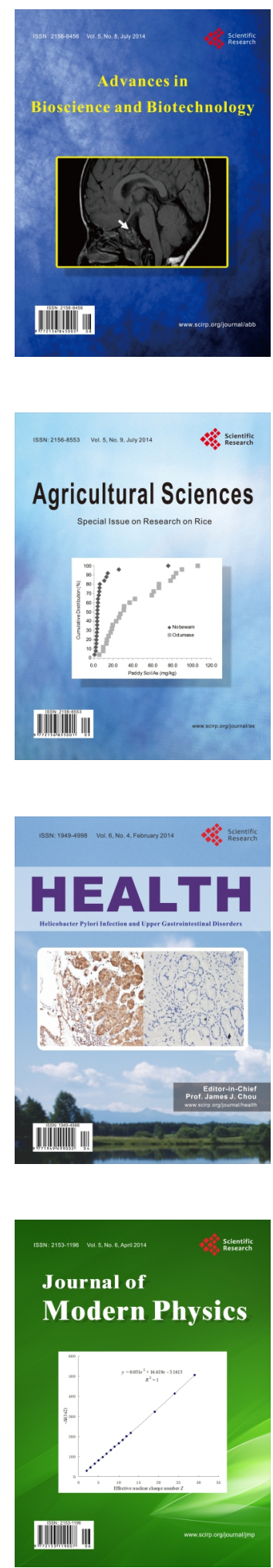
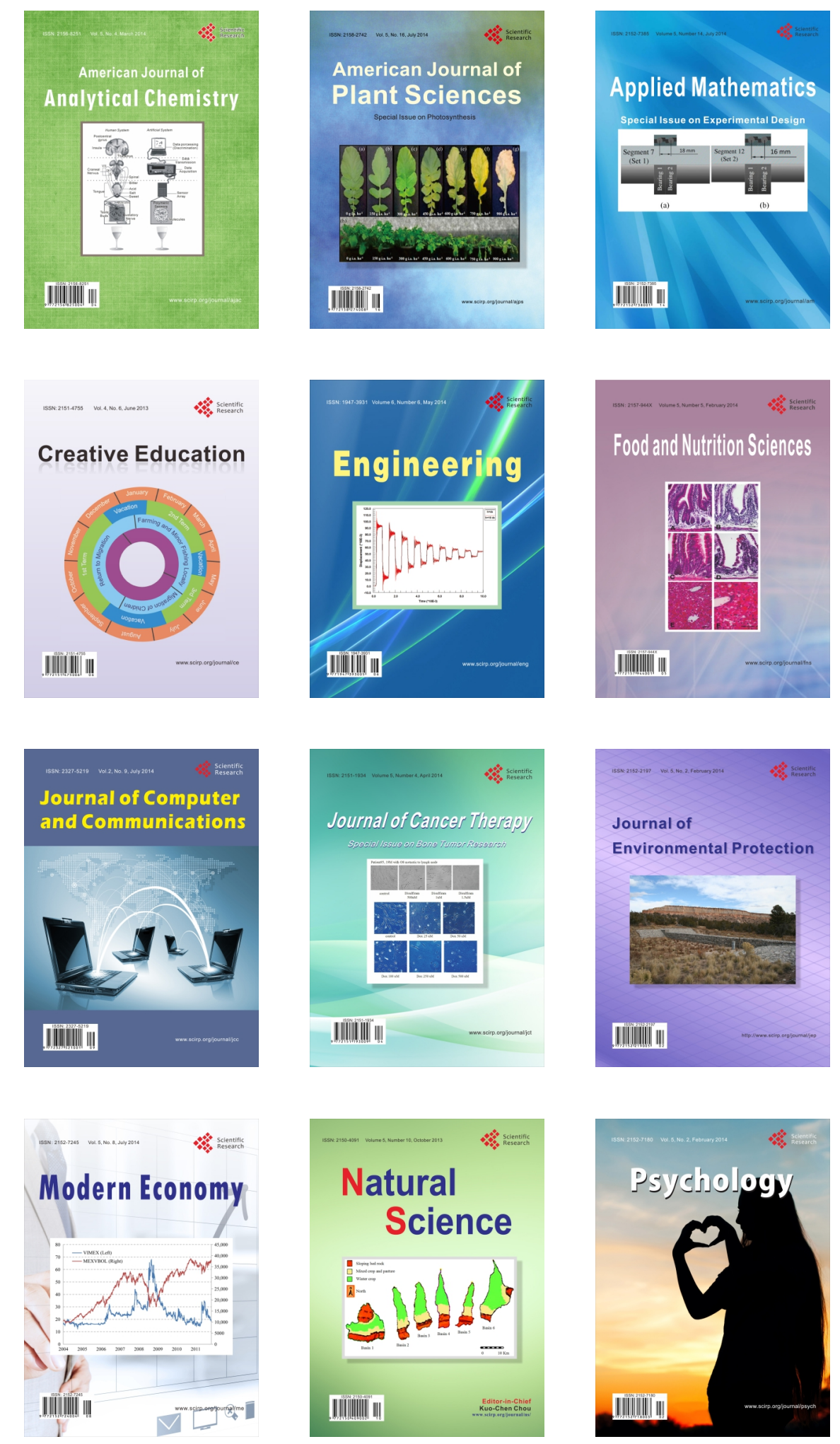